\title{
THE 19TH-CENTURY TRADE IN SWAN SKINS AND QUILLS
}

\section{STUART HOUSTON and MARY I. HOUSTON, 863 University Drive, Saskatoon, SK. S7N 0J8, and HENRY M. REEVES, 22250 Boulder Crest Lane, SE, Amity, OR. 97101}

In an attempt to learn more about the pre-settlement numbers of swans in what are now the three prairie provinces, we consulted the records in the Hudson's Bay Archives (HBCA), Provincial Archives of Manitoba, in Winnipeg. Much has been written about the economics of the fur trade in what is now Canada, but little attention has been paid to one important side item of trade, swan skins and swan and goose quills. Few naturalists have realized what prodigious quantities of these two items were shipped from Hudson Bay back to Britain, as an additional item of trade along with valuable furs. Such overharvesting, superimposed on subsistence use, no doubt contributed to the Trumpeter Swan's decline in numbers and range, and to some decline in the Tundra Swan as well.

Samuel Hearne, who founded Cumberland House [Saskatchewan] in 1774, the first inland trading post of the Hudson's Bay Company (HBC), later reported that the Indians killed Trumpeter Swans "in such numbers that the down and quills might have been procured in considerable quantities at a trifling expence; but since the depopulation of the natives by the small-pox ... no advantage can be made of those articles, though of considerable value in England." Hearne also noted that one Trumpeter Swan egg was "a sufficient meal for a moderate man, without bread, or any other addition." ${ }^{10}$ One thirty-pound $(14 \mathrm{~kg})$ swan, the heaviest bird in North America, provided a great deal of food for hungry people, especially welcome in spring after a winter diet of fish and pemmican. Hearne also reported that the swan skins "of which the Company have lately made an article of trade," became a trade item only near the end of the 18 th century. After quoting from Hearne, Oliver Goldsmith in 1840 wrote: "They are much sought after ... for their flesh, their quill-feathers, and their down." Since The Fur Trade in Canada: An Introduction to Canadian Economic History ${ }^{12}$ apparently does not mention and certainly fails to index swan skins, our search led elsewhere.

How common was the Trumpeter, east of the Rockies, in the 1600s? By extrapolating from the 1968 density of one swan per $20 \mathrm{~km}^{2}$ in Alaska, and projecting this over an area of 2.6 million $\mathrm{km}^{2}$ of the potential 4 million $\mathrm{km}^{2}$ of prairie and boreal forest east of the Rocky Mountains, Lumsden made a credible estimate for 1600 A.D., really a "best guess" of 130,000 Trumpeter Swans. ${ }^{13} \mathrm{He}$ felt that annual removal of 3000 to 5000 swans from this population for swan skins, possible only after the advent of firearms, "would not have been an excessive harvest." Such analysis of course presumes no drop in population over two or more centuries. There is good evidence that swans were common at Moose Factory in 1674 , but the swan flight into James Bay had almost disappeared 
by $1783-85 .{ }^{13}$ Whether or not Lumsden's early population estimates are credible, by the 1913 meeting of the American Ornithologists' Union there were predictions that "this magnificent bird was nearing extinction; and would soon disappear forever." Henry Coale could find, in a survey of all museums, only sixteen specimens collected between 1856 and 1909, five of them from Canada, preserved with authentic data. E.S. Cameron reported in a letter to Coale on 30 April 1914: "Twenty years ago Trumpeter Swans were common in [north-eastern] Montana, and used regularly to winter here, but are now on the verge of extinction."3 By 1935, only 69 Trumpeter Swans were known to exist in the wild, but unrecorded flocks also inhabited parts of Alaska and the Grande Prairie region of Alberta. $^{16}$

The Trumpeter was not the only swan to be affected. The Tundra Swan disappeared as a breeding species from the general area of Hudson Bay for over 150 years, from before 1800 through 1969; they have since returned to breed in northern portions of Manitoba, Ontario and Quebec. $^{13}$

\section{Swan Skins}

The number of swan skins listed for sale in London, 1799-1913, increased from a low of 168 in 1804 to 4305 in 1813. The different number of skins must have represented an increase in interest or value or both, rather than a change in the numbers or availability of swans of both species. The peak years were 1826 , $1827,1830,1834$, and 1837, with $5817,5052,5636,7918$ and 6600 swan skins, respectively, sold in those years, the five highest on record (HMR from HBCA, Figure 1). The last time numbers were over
1000 was in 1850 , with 1038 skins. Average annual numbers for the decades ending in $1820,1830,1840$, $1850,1860,1870,1880$ and 1890, respectively, were $2735,3379,3876$, $1897,981,627,190$, and 120 . The final recording was of 108 swan skins in 1891 (HMR).

These figures are somewhat at variance with and only loosely correspond to those obtained by Roderick Ross MacFarlane, from the Hudson's Bay fur catalogues "for sale in London." From 1853 to 1877 the HBC sold a total of 17,671 , or an average of nearly 707 skins a year. ${ }^{15}$ The HBC catalogues listed seven good years (1853 to 1856, 1861, 1862 , and 1867), with sales ranging in those years between 985 and 1,312 , the maximum reached in 1854. There were seven poor years (1870 to 1877), with returns varying between 338 and the minimum of 122 in $1877 .{ }^{15}$

MacFarlane also provides helpful details concerning the major sources of these skins. From 1854 to 1884 , inclusive, Athabasca District turned out 2,705 swan skins, nearly all of them from Fort Chipewyan. Mackenzie River District supplied 2,500 skins from 1863 to 1883 . From 1862 to 1877 Fort Resolution, Great Slave Lake, contributed 798. For 1889 Athabasca traded but 33 , as against 251 skins in 1853. In 1889 and 1890 , Île-à-la-Crosse, headquarters of the English River District, sent out only two skins each year. ${ }^{15}$ Why were these large swans rarely seen by explorers and traders passing through? One presumes they retreated to large marshes and small lakes for breeding and moulting, off the path of those travelling by river canoe routes, and bypassed by those travelling overland. 


\section{HBC Swan skin annual totals}

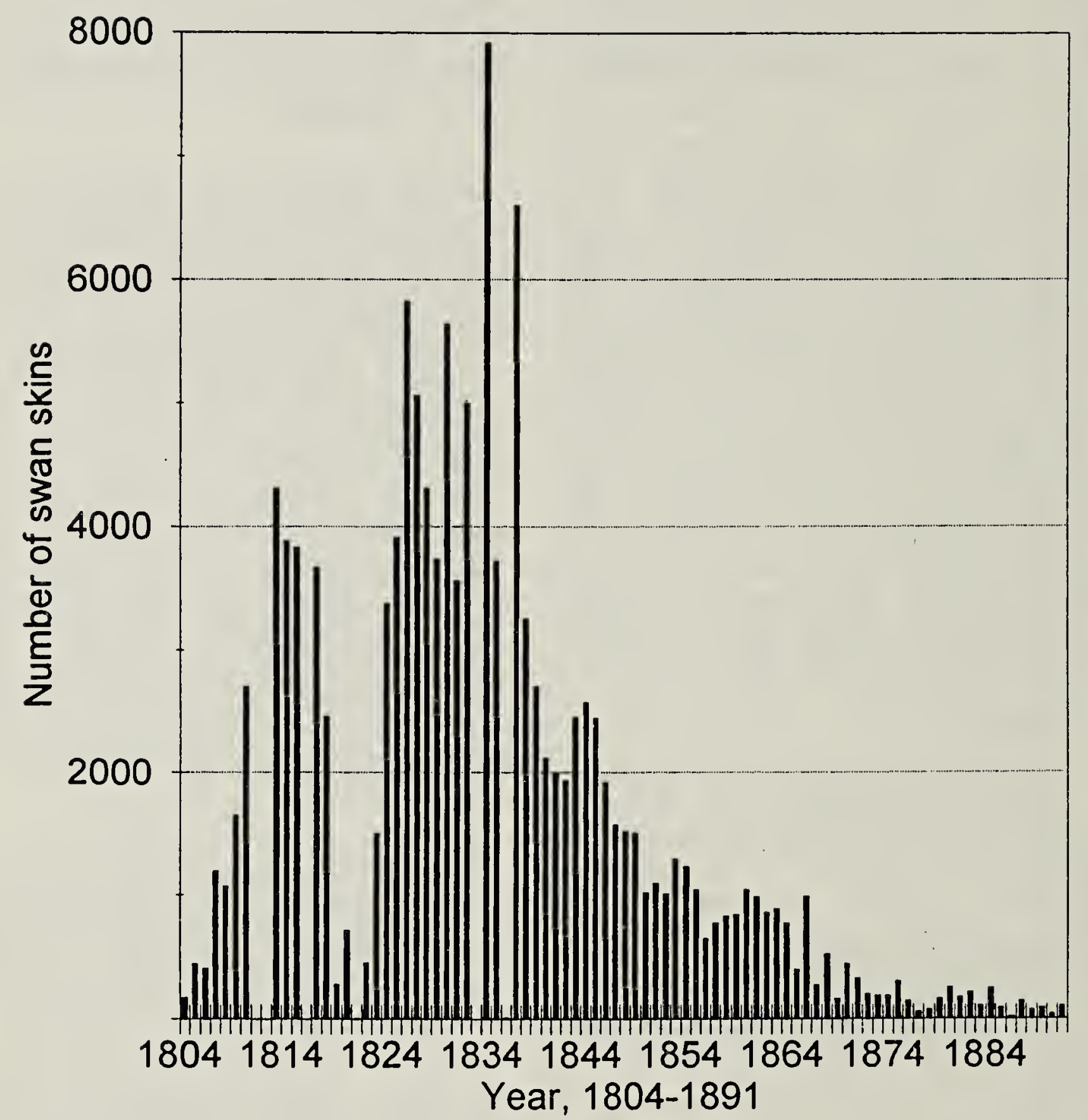

Figure 1. Swan skin annual totals, 1804-1891. Listed for sale by the Hudson's Bay Company, London. Compiled by H.M. Reeves from A.53/1, the Fur Trade Importation Book, 1719-1912. Hudson's Bay Company Archives, Provincial Archives of Manitoba (HBCA, PAM).

There is local corroboration from Fort White Earth \#1, on the banks of the North Saskatchewan River south of the present town of Smoky Lake, Alberta. Alexander Henry the Younger, immediately after building this fort, reported that his men brought 70 swan skins from nearby Smoky Lake on 23 July 1810, and that eight days later he had 208 swan skins in stock. ${ }^{4}$ Cam Finlay reports that at Fort Edmonton, the only source we know of that specifically identified quills as being from swans, 810 swan skins and 460 swan quills were collected in 1810-11, 1206 skins and 450 quills in 1811-12, and 1316 skins and 2740 quills in 1812$13 .^{7}$ All other listings of quills may well have been a combined total of both goose and swan quills. Archaeological excavations have confirmed the presence of Trumpeter Swan bones from Fort White Earth. ${ }^{11}$ 
Table 1. SWAN SKINS FROM HUDSON BAY LISTED FOR SALE, 1804-1819

\begin{tabular}{|c|c|c|c|c|c|c|c|}
\hline Year & Churchill & $\begin{array}{c}\text { York } \\
\text { Factory }\end{array}$ & Severn & Albany & Moose & Eastmain & $\begin{array}{c}\text { Sum } \\
\text { Six } \\
\text { Posts } \\
\end{array}$ \\
\hline 1804 & & 168 & & 37 & & & 205 \\
\hline 1805 & & 435 & & & & & 435 \\
\hline 1806 & & 396 & & & & & 396 \\
\hline 1807 & 28 & 1133 & & 27 & & 4 & 1192 \\
\hline 1808 & 9 & 997 & 1 & 60 & & & 1067 \\
\hline 1809 & 6 & 1576 & & 70 & & & 1652 \\
\hline 1810 & & 2706 & & & & & 2706 \\
\hline 1811 & & & & & & & 0 \\
\hline 1812 & & & & & & & 0 \\
\hline 1813 & 219 & 4066 & & 4 & & 16 & 4305 \\
\hline 1814 & & 3853 & & & 16 & 15 & 3884 \\
\hline 1815 & 348 & 3487 & & & & & 3835 \\
\hline 1816 & & & & & & & 0 \\
\hline 1817 & & 3666 & & & 4 & & 3670 \\
\hline 1818 & & 2462 & & & 1 & & 2463 \\
\hline 1819 & & 273 & & & 6 & & 279 \\
\hline Total & 610 & 25218 & 1 & 198 & 27 & 35 & 26089 \\
\hline
\end{tabular}

Questions remain as to which species was killed for the swan skin trade, Trumpeter or Tundra (Whistling) or both? Where were they taken? How many were taken only in spring or fall migration? How many in summer, before the immatures could fly and during the adult flightless summer moult? What price did they fetch? What were these swan skins used for? Partial answers require melding of information from archaeological sources, fur trade archives and published historic records, but complete answers are no longer possible.

1) Both species were taken, the Tundra Swan only in migration.

2) A breakdown by individual trading posts on Hudson Bay, compiled by HMR, is available for 1804 through 1819 (Table 1). Almost $97 \%(25,218$ of 26,089 swan skins) came through York Factory, the direct link with the plains and parkland areas of what are now the prairie provinces, far south of the nesting area of the Tundra (Whistling) Swan.
3) For the subsequent two decades, 1821 through 1841, numbers of swan skins taken in each trading district have been transcribed from HBCA B239/h/1 by MIH ( Table 2). The first four areas, in descending order of importance, were the Saskatchewan River (Carlton and Edmonton, which traded with Indians on the plains), Churchill River (then called English River), Athabasca, and the Swan River. Each of these localities was south of the nesting area of the Tundra Swan. With the exception of 1804, these figures correspond exactly with HMR's numbers for sale in London for those years. These inland localities delivered their furs to York Factory, where in the previous two decades these skins would have been listed as from York Factory.

4) A scattering of early historical nest records confirm that Trumpeter Swans bred on the northern Great Plains from lowa up through North Dakota into Manitoba and Alberta, north into the parklands and 


\begin{tabular}{|c|c|c|c|c|c|c|c|c|c|c|c|c|c|c|c|c|c|c|c|}
\hline & $\begin{array}{l}\text { ज्ञ } \\
\text { 음 }\end{array}$ & 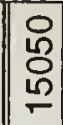 & 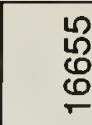 & & $\begin{array}{l}\text { W } \\
\text { ల్ }\end{array}$ & 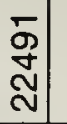 & $\begin{array}{l}8 \\
\mathbb{8} \\
6\end{array}$ & 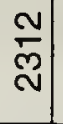 & $\stackrel{g}{\circ}$ & $\stackrel{N}{N}$ & 足 & 은 & $\stackrel{\mathscr{N}}{\stackrel{N}{*}}$ & 10 & \begin{tabular}{l|l}
8 \\
\%
\end{tabular} & $\begin{array}{ll}\overrightarrow{5} \\
\stackrel{5}{5}\end{array}$ & 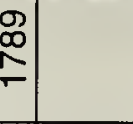 & $\begin{array}{l}\text { న } \\
\text { M } \\
\frac{N}{N}\end{array}$ & \\
\hline & $\underset{\Phi}{\sigma}$ & 8 & $\frac{0}{m}$ & & $\stackrel{m}{\sim}$ & $\begin{array}{c}- \\
q \\
q\end{array}$ & $\stackrel{\infty}{\mp}$ & : & & & & $\stackrel{\infty}{-}$ & - & & & 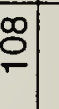 & & 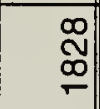 & \\
\hline & $\stackrel{\text { 요 }}{\stackrel{\infty}{\circ}}$ & 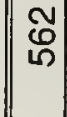 & 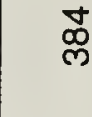 & & $\hat{m}$ & $\begin{array}{l}\mathscr{P} \\
\text { ñ }\end{array}$ & ஜㅁ & $\bar{\Omega}$ & & & & 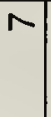 & (n) & & & 苟 & & $\begin{array}{l}\text { 吕 } \\
\stackrel{2}{\sigma}\end{array}$ & \\
\hline & 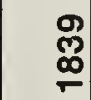 & $\frac{0}{1}$ & 号 & & $\stackrel{N}{\forall}$ & $\stackrel{m}{\tilde{q}}$ & $\stackrel{\bullet}{\oplus}$ & 요 & & & & 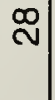 & $\lambda$ & & & $\mathcal{F}$ & & $\begin{array}{l}\text { Dे } \\
\text { iे }\end{array}$ & \\
\hline & 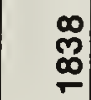 & $\begin{array}{l}\mathscr{p} \\
\end{array}$ & ర్ & & $\bar{m}$ & $\begin{array}{l}\infty \\
\mathbb{N} \\
\mathbb{0}\end{array}$ & 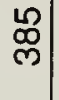 & f & & & & $\tau$ & - & & & 怘 & & 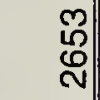 & \\
\hline & $\underset{\mathscr{\infty}}{\boldsymbol{\sigma}}$ & 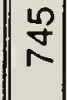 & 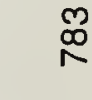 & & 命 & ָొ & প্ণ & $\stackrel{\text { 。 }}{\circ}$ & & & & $\stackrel{m}{-}$ & - & & & 8 & 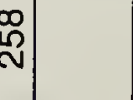 & $\begin{array}{l}\text { D. } \\
\text { जै }\end{array}$ & \\
\hline & $\begin{array}{l}\mathscr{\infty} \\
\stackrel{\infty}{\leftarrow}\end{array}$ & $\begin{array}{c}0 \\
N\end{array}$ & 命 & & $\bar{\lambda}$ & ઠे) & $\begin{array}{l}\infty \\
\stackrel{\infty}{\sigma}\end{array}$ & $\begin{array}{l}0 \\
0 \\
m\end{array}$ & & & & $\infty$ & $\nabla$ & & $\stackrel{\infty}{\leftarrow \mid}$ & & 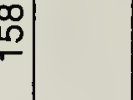 & 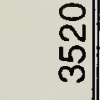 & \\
\hline & $\stackrel{\mathscr{\infty}}{\infty}$ & m & ర్ల్ర & & $\bar{m}$ & $\begin{array}{l}\hat{0} \\
0 \\
0\end{array}$ & \begin{tabular}{l}
$\infty$ \\
$\infty$ \\
\multirow{q}{*}{}
\end{tabular} & $\stackrel{\sim}{\underset{N}{N}}$ & & & & 9 & $N$ & & $\nabla$ & 요요 & $\underset{q}{\infty}$ & $\frac{0}{\frac{0}{m}}$ & \\
\hline & 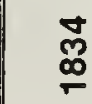 & $\frac{0}{2}$ & 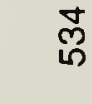 & & $\stackrel{\infty}{\stackrel{\infty}{\Sigma}}$ & $\begin{array}{l}\text { f } \\
\dot{\sigma}\end{array}$ & 今̂ & $\begin{array}{l}\mathscr{D} \\
\stackrel{N}{N}\end{array}$ & & & & $\mp$ & n) & & 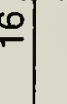 & & & $\frac{8}{m}$ & \\
\hline & 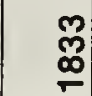 & $\mid$\begin{tabular}{|l}
$\mid 2$ \\
0 \\
0 \\
0
\end{tabular} & సે & & $\begin{array}{l}\mathscr{\Xi} \\
\stackrel{2}{2}\end{array}$ & $\frac{0}{0}$ & 总 & $\mathscr{8}$ & $m$ & & & 이 & $\mp$ & & 0 & tot & & 怘 & \\
\hline & $\underset{\tilde{\infty}}{\widetilde{2}}$ & 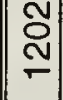 & $\stackrel{\infty}{\stackrel{0}{+}}$ & & $\begin{array}{l}0 \\
\stackrel{2}{*}\end{array}$ & ণ్ & 各 & षे & & & & 이 & $\mp$ & 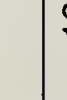 & 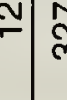 & ले & & $\begin{array}{l}\text { जิ } \\
\text { ஸे }\end{array}$ & \\
\hline & - & 怘 & 告 & & โ) & 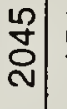 & 点 & $\stackrel{ \pm}{N}$ & $\stackrel{\mathscr{N}}{\mid}$ & $\stackrel{\infty}{\sim}$ & & - & $\stackrel{\nabla}{\nabla}$ & & $=$ & $\stackrel{\Omega}{\mathrm{n}}$ & & 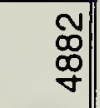 & \\
\hline & $\underset{\mathscr{\infty}}{\stackrel{\infty}{\leftarrow}}$ & $\pi$ & $\stackrel{\infty}{\stackrel{2}{\wedge}}$ & & 9 & 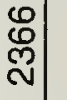 & & $\stackrel{\mathscr{T}}{-}$ & ล & 0 & & - & 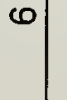 & 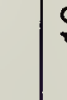 & $\stackrel{8}{9}$ & : & vै & $\begin{array}{c}\text { స్ల } \\
\text { N }\end{array}$ & \\
\hline & $\underset{\infty}{-\infty}$ & $\frac{m}{N}$ & $\underset{\widehat{N}}{\underline{N}}$ & & 守 & $\begin{array}{l}8 \\
0 \\
-\end{array}$ & $\stackrel{\mathscr{m}}{\leftarrow}$ & $v$ & $m$ & 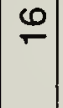 & & & 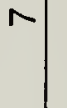 & & छू & & & 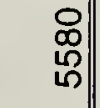 & \\
\hline & జ్జ & $\mid \begin{array}{l}\infty \\
\substack{n \\
N}\end{array}$ & $\stackrel{\text { D }}{=}$ & & ㅇ & $\begin{array}{l}n \\
\square \\
\square\end{array}$ & ๙ิ & $\infty$ & $\infty$ & $\stackrel{\sim}{\sim}$ & & $\nabla$ & $\nabla$ & & 8 & 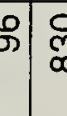 & D. & $\begin{array}{l}0 \\
o \\
g \\
\forall\end{array}$ & \\
\hline $\mathcal{Y}$ & 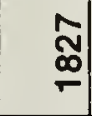 & $\frac{m}{\frac{m}{f}}$ & ৪ & & స్ స్ & $\stackrel{n}{\stackrel{n}{N}}$ & f & & & $\cong$ & - & $N$ & - & $c$ & $\begin{array}{l}0 \\
0\end{array}$ & & & $\frac{\bar{\sigma}}{\bar{\nabla}}$ & \\
\hline a. & 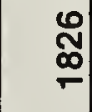 & 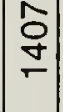 & $\stackrel{\mathbb{a}}{\underset{N}{N}}$ & & 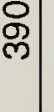 & 冬 & $\begin{array}{l}n \\
m \\
m\end{array}$ & ? & & 尺 & & & 으 & 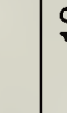 & 0 & ৪। & & 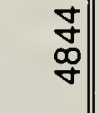 & \\
\hline$\frac{\omega}{z}$ & $\underset{\mathbf{\infty}}{\leftarrow}$ & $\begin{array}{l}\mathbf{y} \\
\text { t } \\
\end{array}$ & ஓ & & 芯 & '্) & ৪) & : & & $\hat{\mathrm{N}}$ & & 10 & $\stackrel{d}{\stackrel{d}{*}}$ & $\xi$ & $\stackrel{乛}{*}$ & & & $\begin{array}{l}\stackrel{+}{0} \\
\stackrel{\sigma}{\sigma}\end{array}$ & $\frac{\alpha}{a}$ \\
\hline$<$ & $\underset{\mathbb{\Phi}}{\sim}$ & & ํㅗㅇ & & ָิ & 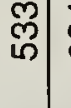 & స్రు & 8 & & ले & & $\nabla$ & $\sim$ & & e & 5 & & $\begin{array}{c}0 \\
\text { ô. } \\
\text { N }\end{array}$ & $\underline{\underline{\mathbf{m}}}$ \\
\hline$\frac{0}{2}$ & $\underset{\widetilde{\infty}}{\stackrel{\mathscr{N}}{\sim}}$ & & 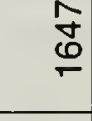 & & $\stackrel{\simeq}{\mp}$ & \begin{tabular}{l}
$\infty$ \\
$⿱ 亠 乂$ \\
\hdashline
\end{tabular} & $\underset{8}{\infty}$ & $n$ & N & 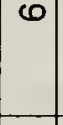 & & $N$ & & $m$ & $\stackrel{a}{*}$ & क⿻ & & $\begin{array}{l}\text { : } \\
\text { ¿ }\end{array}$ & ్ָ \\
\hline$\vec{w}$ & 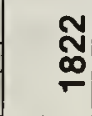 & 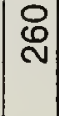 & 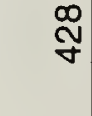 & & 足 & 只 & :్రి & ㅇ & $\stackrel{\infty}{q}$ & $\infty$ & & $N$ & 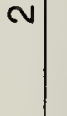 & & & 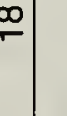 & & $\begin{array}{l}\mathbb{V} \\
\mathbb{T} \\
\mathcal{T}\end{array}$ & 흔 \\
\hline 5 & $\underset{\infty}{\stackrel{\infty}{-}}$ & & in & & $\stackrel{\sim}{\sim}$ & $\begin{array}{l}0 \\
\stackrel{0}{N} \\
\stackrel{2}{2}\end{array}$ & ले & $\neq$ & 의 & & $\hat{m}$ & & & & & & & mิ & 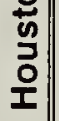 \\
\hline 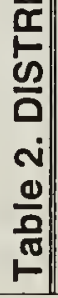 & 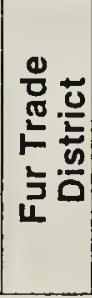 & 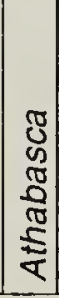 & 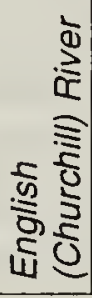 & 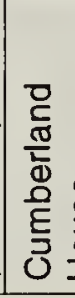 & & 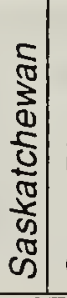 & 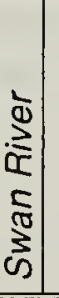 & 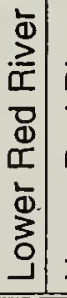 & 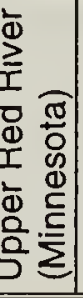 & 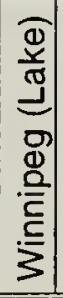 & 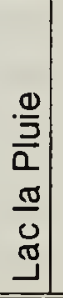 & 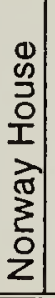 & 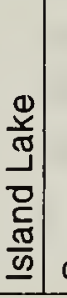 & 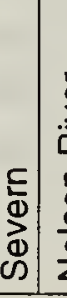 & 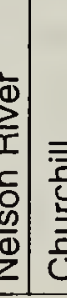 & 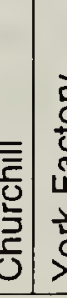 & 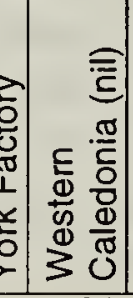 & 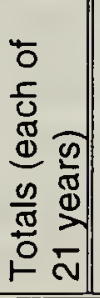 & 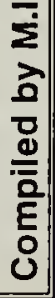 \\
\hline
\end{tabular}


southern mixed forest. ${ }^{1}$ Lumsden suggests that this larger species needed an ice-free period of at least 140 days, and preferably 154 days, to complete its long breeding cycle, coinciding nicely with what we know about the northern edge of their presumed breeding, south of that of the smaller Tundra Swan. ${ }^{13}$

5) The most authoritative comment is by Dr. John Richardson, who collected birds at Cumberland House and Carlton House in Saskatchewan in the 1820s; he reported that "It is to the Trumpeter that the bulk of the Swan-skins imported by the Hudson's Bay Company belong." As late as 1859 , Blakiston concurred that the Trumpeter was still the commoner species at Carlton. ${ }^{2}$

6) Harry Duckworth (pers. comm.) has recorded purchases in London: in January 1808, Joseph Binter purchased $£ 482$ worth of swan skins; George Smith, £227; Peter Raymond Poland, £163; and Schnerot (?), £85. In April 1810, Joseph Binter purchased £195 and Mr. Riechard, $£ 100$. If we make a wild assumption that these men purchased all of the 1192 and 1652 swan skins sold in each of those years for the European market, then each swan skin may have been worth nearly $£ 1$ in the first instance and one-fifth this amount two years later. If these businessmen purchased only half the swan skins, the amount per skin would double, and if only one-fifth, then the unit value would increase by five times.

7) Wilmore tells us that in Europe swan skins were used in the manufacture of powder puffs for women. ${ }^{21}$ One wonders, with the large numbers involved, whether the skins might also have been used for coat linings? Harold Burgess, a researcher into Trumpeter Swans, has read in historical fiction that swan skins were also used for making vests, ceremonial robes and for ornaments such as epaulets on uniforms of high-ranking officials. Andrew Dawney ${ }^{5}$ tells us swan skins were still valued at five pence (25 cents) a pelt in 1899. The beautiful snow white down of the Bewick's Swan, when dressed by a furrier, made women's neck pieces (boas) "of univalled beauty." Warwick, Pitz and Wyckoff (1965) illustrate a loose jacket, brought over from Holland by Dutch settlers in New York, "trimmed with fur or swansdown around the neck, down the front, and around the bottom." 20 In Russia the tough skin and warm soft pelt was used for wallets, jackets and caps. Barbara Nichols ${ }^{17}$ reports that swan skins were used for powder puffs, quilts, pillows and mattresses. Jack London, in his short story, "The nightborn" in Jack London Short Stories, mentioned a robe of swan-skins" (Karen Lunsford, pers. comm.). In Little House on the Prairie, $\mathrm{Pa}$ Ingalls shot a swan and $\mathrm{Ma}$ Ingalls made a small swan cape for the youngest girl (Brian Burchett, pers. comm.).

The demand for swan skins was no respecter of species. Earlier in this century, Frank M. Chapman found "hundreds of thousands" of Black-necked Swan skins in an Argentina warehouse awaiting shipment to be made into women's powder puffs. ${ }^{5}$

Judith Hudson Beattie has allowed us to reproduce the official Hudson's Bay Company directive that told traders how to prepare swan skins of the highest value (Figure 2). This printed "broadside" dated from circa 1817 , when R. Causton and Son had their office at 21 Finch Lane (Harry Duckworth, pers. comm.). Nearly a 


\title{
DIR ECTIONS \\ FOR \\ $C U \mathbb{R} \mathbb{I} N G$ AND PRESERIING \\ Swan Skins.
}

\begin{abstract}
As Swans are gencrally shot, let the Feathers be pulled out while the Bird is hot, with the greatest care not to injure the Fine Down underneath; the Blood that may be upon the Skin may be washed off with Soap and Water, and well dried afterwards; the Bird will be skinned much easier after the Feathcrs are pulled, and must be done in this manner,-
\end{abstract}

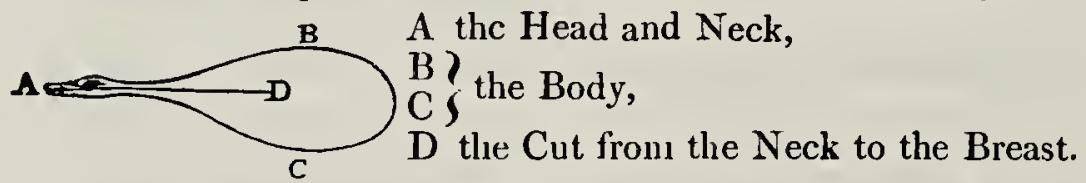

The Back must not be cut, and it is not necessary, as the Bird may be skinned by drawing it through the Part cut from the Head to the Breast; when dry, it may be turned the Pelt or Skin outside, which will protect the Down from being injured by Grease, \&c. and will come safe in Packages any distance.

N.B. The Swan Feathers should not. be mixed with those of the Goose.

Frinted by R. Caunton \& Son, 21 , Finch-Lase, Corrhill, London.

Figure 2. Directions for curing and presenving swan skins [ca 1817]. HBCA A.63/22 fo. 3 (N13516). Courtesy Judith Hudson Beattie and HBCA, PAM.

half-century later, the Governor and Committee gave very similar instructions to Ferdinand Jacobs on 12 May, saying "We are informed that the skin of the Wild Swan may probably turn out of some utility in our trade ..."(HBCA A.6/11, fo 170d).

\section{Swan and Goose Quills}

The flight feathers of all birds were long known as quill-feathers. Some clues to the increasing interest in quills as a commercial item derive from entries in the 1942 edition of Encyclopedia Brittanica. Under Feather: "The earliest period at which the use of quill feathers for writing is recorded is the 6th century ... Only the five outer wing feathers of the goose are useful for writing, and of these the second and third are the best, while left-wing quills are more esteemed than those of the right as they curve outward and away from the writer using them. ... Swan quills indeed are better than those from the goose." ${ }^{14}$ Under Pen:
"In 1809 Joseph Bramah devised and patented a machine for cutting up the quill into separate nibs by dividing the barrel into three or even four parts, and cutting these transversely into two, three, four and some into five lengths." 6 Under Bramah: "Joseph Bramah, 17481814, was an English engineer and inventor, who invented the hydraulic press, paper-making machinery, a numerical machine for printing banknotes, and the Bramah lock."

Until improved nibs were invented, quills required continual sharpening. The average clerk would use more than one new quill pen per day. "Bed-feathers" were first sold by the $\mathrm{HBC}$ in the London market in November 1736 and 'goose quills' were first offered in December 1744 and November 1745 (Harry Duckworth, pers. comm.). Swan quills were in greater demand and sold (in bundles of 25 or 100 ) at the highest price. Swan and goose quills from Hudson 


\section{HBC Quill Annual Totals}

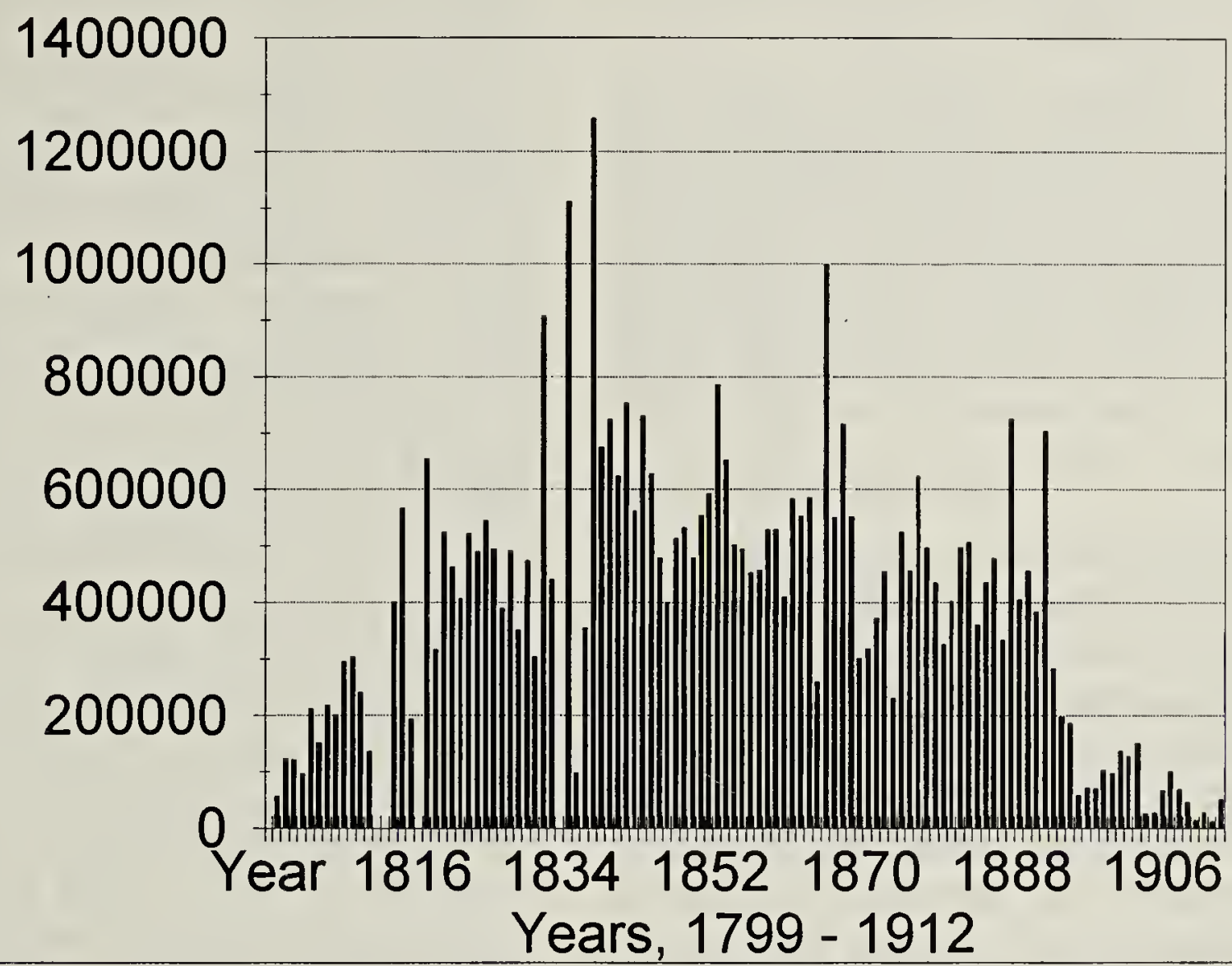

Figure 3. Goose and swan quill annual totals, 1799-1911. Listed for sale by the Hudson's Bay Company, London. Compiled by H.M. Reeves at HBCA, PAM

Bay sold in increasing numbers, from 58,000 in 1799 to 566,632 (think of the tedium involved in counting them!) in 1814 and 655,030 in 1817 (HMR from HBCA, Figure 3). Sales peaked at $1,112,000$ in 1834 (the year that a grand total of $18,732,000$ quills were sold in London ${ }^{7}$; and $1,259,000$ in 1837 . At a maximum of ten useable quills per bird, 1837 saw the sacrifice of over 100,000 swans and geese.

There is only a modest correlation between the number of swan skins and the number of quills sold in a given year. The climate was colder then than now, and sailing ships each year ran the gauntlet of icebergs in Hudson Strait. The four years when no skins and no quills were sold in London were years when the annual ship or ships were unable to return to England, and each gap was followed by an abnormally high total. The first gap of two years may be explained because the Edward and Ann, carrying the first 105 Selkirk settlers bound for Red River, was too pressed to make the customary stop at Churchill. The 1811 voyage, taking 61 days, set an all-time record. In 1812, the problems of war with the United States may have been reflected as far north as Hudson Bay. In 1816 the Prince of Wales was caught in ice and did not return to England and in 1833 the Prince Rupert $V$ suffered a similar fate. Extremely low numbers of skins and low-average numbers of quills in 1836 perhaps resulted from two of the three ships that year having to winter in the bay, while the Prince 
Rupert $V$, after being beset in ice from 23 August to 19 September, returned without fully unloading its inbound cargo.

In general, sales of quills and swan skins both peaked in the 1830 s, but quantities of the prime "Hudson Bay quills" presumably came from geese. Quill numbers did not fall off as quickly as did swan skins, and remained relatively high through 1891.

Finlay's informative book, The History of the Quill Pen, ${ }^{8}$ shows that at one time, crow quills, for architectural drawing, fetched as much as 9 shillings per 100; turkey quills, for law writing, were 7 shillings; domestic goose quills, 15 shillings; "Hudson's Bay quills" and swan quills each went as high as 63 shillings per 100 . In England, huge flocks of geese were farmed mainly for their quills; in 1812 nine million geese were plucked for the domestic market. The quills of the wild geese from North America were even more highly regarded, since "the best quills came from the coldest countries." Swan quills were even better, "a single swan outlasting as many as fifty made from goose quills." The five largest feathers from each wing were used. The first primary was called a pinion; the second and third were labelled "seconds" and the fourth and fifth, "thirds." 8 There was a tradition that swans' quill pens were left full feather. $^{8}$

Quills were obviously big business; 27 quill and pen manufacturers and dealers were listed in Pigot's London Directory (1822), while Newcastleupon-Tyne had three. Though metal pens became available in the 1820s, most writers and almost all lawyers preferred the quill, as it "enables an expert Scribe to both text and en- gross in a better style, and to cut the letters more clearly." 8 The last large year for quills from Hudson Bay was 1865 , when the total was just one thousand short of the million mark. Numbers dropped to 59,000 in 1895 , reflecting increasing competition from the development of metal pen nibs. In the last two years on record, 1911 and 1912, 12,000 and 52,000 quills, respectively were sold (Figure $3)$. Manufacture of metal pen nibs became common in the United States in the 1860s, but it is apparent that not all writers switched for another half-century. As late as 1894 , one firm supplied the India Office with more than two million quill pens, and in 1908 swan pens were sold with gold- and silver-plated tips. The last quill company closed its offices in London in 1954, when computer "punched card systems" were now in evidence! ${ }^{8}$

\section{Swans as Food}

In medieval England ownership of swans was a mark of social standing. As a food item, especially as the set-piece for banquets, a swan was extremely expensive, selling in London in 1274 for three shillings (36 pence), compared to five pence for a goose and four pence for a pheasant. The punishment for stealing swan eggs was imprisonment for a year and a day. For stealing a swan, the thief had to pour wheat over the suspended bird, hung by its beak, until the tip of the beak was covered; the wheat was then paid to the swan owner. $^{5}$

\section{Trumpeter Swan Populations in North America}

Bones from early historic sites suggest that the Trumpeter Swan suffered from more drastic declines than other large birds such as the Sandhill and Whooping Cranes. All 
these species fared poorly with settlement, and were driven out as land was ploughed and marshes drained. Trumpeters began their decline at least a century earlier than the cranes. Originally breeding from at least Kentucky and from Chesapeake Bay north to the maritime provinces and wintering south to northern Florida, Trumpeters quickly disappeared from eastern North America as humans advanced inland. ${ }^{19}$ Undoubtedly subsistence taking of its eggs and meat as prime food items antedated recorded history, but later demand for its skins and quills added to its demise. As Banko summarized in his classic monograph in 1960, "The effects of such exploitation on the far-flung breeding populations of this species for more than 125 years must have been devastating and largely responsible for its extermination over vast regions, particularly in the heart of its Canadian breeding range."

We strongly suspect that the pre1900 falling-off in numbers of Canadian swan skins sold each decade in London reflected over-harvesting of these large birds, aggravated by collecting of both the swans and eggs in season for food, especially after bison numbers fell drastically in midcentury.

Extinction of the Trumpeter Swan was narrowly averted at the turn of this century. With protection, numbers have increased in a gratifying manner, such that the Pacific Coast population in Alaska increased to 9500 individuals by 1991 , while the Rocky Mountain population (including northern Alberta) increased eleven-fold to 2200 individuals. The restored interior population had 629 free-flying birds by $1993 .{ }^{16}$ Trumpeter Swan reintroduction programs are gaining speed wherever they have been attempted; in southern
Ontario they began in 1982. On 1 September 1995 there were 82 Trumpeters flying free and one year later, 123 (Harry Lumsden, pers. comm.). Trumpeter Swan's recovery has been sufficiently successful that it has been de-listed from the federal endangered species list. However, we should learn from the history of its exploitation there are better methods to manage our wildlife resources.

\section{Acknowledgements}

We thank Judith Hudson Beattie, keeper of the Hudson's Bay Archives, Provincial Archives of Manitoba, for continued help and support, and for providing the print of the broadside giving directions for preparation of swan skins. Harold Burgess found two sources of information concerning the use of swan skins. Ruth E. Shea and Rod C. Drewein provided constructive criticism of an earlier draft. Harry Duckworth provided dates for operation of the $R$. Causton printing firm in London, payments made for swan skins in London, and, on our behalf, posed a question to members of the 18th Century Interdisciplinary Discussion Group on e-mail, which brought helpful responses from Karen Lunsford at the University of Chicago and Brian Burchett at the University of North Dakota. Cam Finlay provided data on swan skins and quills from Fort Edmonton.

1. BANKO, WINSTON. 1960. The Trumpeter Swan. North American Fauna \#63. 214 pp.

2. BLAKISTON, THOMAS. 1863. On birds collected and observed in the interior of British North America [part 4]. Ibis 5:121-155.

3. COALE, H.K. 1915. The present status of the Trumpeter Swan, Olor buccinator. Auk 32:82-90.

4. COUES, E. 1897. New light on the 
earlier history of the Greater Northwest: the manuscript journals of Alexander Henry and David Thompson. Francis P. Harper, New York. Vol. 2, pp. 615-616.

5. DAWNEY, ANDREW. 1972. Exploitation, pp. 167-180 in P. Scott, ed., The swans. Houghton Mifflin, Boston.

6. ENCYCLOPEDIA BRITTANICA. 1942. Bramah, Joseph, 4:25; Pen, 17:459460.

7. FINLAY, CAM. 1997. Animal populations and habitat changes in the contact period on the North Saskatchewan. Proceedings of the Rupert's Land Colloquium, Edmonton, 23-25 May 1994, ed. I.M. MacLaren [in press].

8. FINLAY, MICHAEL. 1990. Western writing implements in the age of the quill pen. Plains Books, Wetheral, Carlisle, Cumbria.

9. GOLDSMITH, OLIVER. 1840. A history of birds. Vol. 3, pp. 1-439, in A history of the earth and animated nature. Archibald Fullarton, Glasgow.

10. HEARNE, SAMUEL. 1795. A journey from Prince of Wales Fort, in Hudson's Bay, to the Northern Ocean, in 1769, 1770, 1771, and 1772. T. Cadell, London.

11. HURLBURT, I. 1977. Faunal remains from Fort White Earth, N.W. Co. 1810-1813. Prov. Mus. Alberta Occas. Paper \#1 (pp. 55-60, 107).

12. INNIS, HAROLD. 1956. The fur trade in Canada: an introduction to economic history. University of Toronto Press, Toronto. $463 \mathrm{pp}$.
13.LUMSDEN, H.G. 1984. The pre-settlement breeding distribution of Trumpeter, Cygnus buccinator, and Tundra Swans, C. columbianus, in eastern Canada. Can. Field-Nat. 98:415-424.

14. ENCYCLOPEDIA BRITTANICA. 1942. Pycraft, W.P. Feather, 9:128131.

15. MACFARLANE, RODERICK ROSS. 1905. Notes on mammals collected and observed in the northern Mackenzie River district... . Proc. U.S. Nat. Museum 28:673-764.

16. MITCHELL, CARL D. 1994. Trumpeter Swan (Cygnus buccinator) in The birds of North America, No. 105 (A. Poole and F. Gill, eds.). Acad. Nat. Sci., Philadelphia, and AOU, Washington, DC.

17. NICHOLS, B. 1990. Trumpeter. Michigan Natural Resources Oct. 1990: 1217.

18. RICHARDSON, JOHN, and WILLIAM SWAINSON. 1831 [=1832]. Fauna Boreali-Americana, Vol. 2, The birds. John Murray, London. 501 pp.

19. ROGERS, PHILIP M., and DON A. HAMMER. 1981. Ancestral breeding and wintering ranges of the Trumpeter Swan (Cygnus buccinator) in the eastern United States. Tennessee Valley Authority, Knoxville, TN.

20. WARWICK, E., H.C. PITZ, and A. WYCKOFF. 1965. Early American dress: the colonial and revolutionary periods. Benjamin Blom, New York.

21. WILMORE, S.B. 1974. Swans of the world. Taplinger Publishing, New York, p. 91.

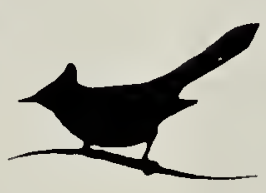

Bird Names

Anna, Duchess of Rivoli (1806-1896), wife of Prince Victor Massena and daughter-in-law to one of Napoleon's marshals, Andre Massena, Duc de Rivoli - Anna's Hummingbird and Rivoli's Hummingbird for Victor. 